\title{
La desestructuración de un barrio industrial en la crisis de la modernidad valdiviana, Chile ${ }^{1}$
}

\author{
Laura Rodríguez N. ${ }^{2}$, Elisa Cordero J. ${ }^{2}$, Gustavo Rodríguez J. ${ }^{2}$, \\ Cristián Gutiérrez ${ }^{2}$
}

\begin{abstract}
RESUMEN
Entender la evolución urbana, como parte de un sistema complejo, es de vital importancia para el desarrollo futuro de la urbe. En este contexto, las denominadas zonas industriales, de sumo interés para la vitalidad y estabilidad de una ciudad, se alteran a causa de cambios paradigmáticos dependientes de modelos político-económicos, emergiendo nuevas y distintivas morfologías urbanas. Esta investigación se desarrolló en un antiguo barrio industrial de la ciudad de Valdivia -Collico-, donde convergen diversos modelos de construcción del espacio urbano. Ilustrativo del auge productivo de una época de fuerte industrialización en el país, y de un posterior decaimiento del área industrial, se ha introducido una serie progresiva de transformaciones, reestructurando la zona antes mencionada.
\end{abstract}

Palabras clave: Espacio urbano, zonas industriales, Valdivia.

\begin{abstract}
The understanding of urban evolution, as a part of a complex system, is crucial to the future of urban development. In this context, the so-called industrial areas, essential to urban vitality and stability, are altered due to paradigmatic changes dependent on economic and political models, creating new distinctive urban morphologies.

This investigation was developed in an old industrial neighbourhood in the city of Valdivia -Collico-, where diverse models of construction of the urban space converge. Illustrative of the productive peak of a period of strong industrialization in the country, and of a subsequent decay of the industrial area, a series of progressive transformations have been introduced and have restructured the zone indicated above.
\end{abstract}

Key words: Urban areas, industrial areas, city of Valdivia.

El fenómeno global de la modernidad fue esencialmente urbano (Kostof, 1992), donde la industria y la mecanización de esta impulsaron una fuerte migración del campo a la ciudad latinoamericana. El caso de Chi-

1 Proyecto DID. S-2005-47, Universidad Austral de Chile. Artículo recibido el 15 de mayo de 2007 y aceptado el 24 de marzo de 2008. le es descrito entre otros autores por Hidalgo (1999) y Borsdorf (2003), y el de la ciudad de Valdivia por Almonacid (2000). La proximidad de la fuerza laboral al aparato productivo, tal como de los dueños de los

\footnotetext{
2 Universidad Austral de Chile (Chile). E-mail: Irodriguez@uach.cl, elisacordero@uach.cl, gustavorodriguez@uach.cl,cristiankaturra@gmail.com
} 
medios de producción al vecindario que albergaba a las instalaciones industriales, expresó un principio, determinando una manera de hacer la ciudad moderna (Cuthbert, 2006).

Harvey (1990) en su libro "La condición de la postmodernidad" ofrece, a partir de varios autores, una conceptualización general de la modernidad fordista, la cual, de acuerdo a él, con sus economías a escala, la intervención del Estado, la sindicalización, y la centralización de la producción, se contrapone con la acumulación flexible del postfordismo posmodernista descentralizado, individualista y con una creciente contraurbanización. A lo anterior Martin (1994) agrega que mientras el paradigma de la postguerra estaba basado en el petróleo de bajo costo, la maquinaria eléctrica, la producción y el consumo en masa, el nuevo paradigma emerge apuntalado por las tecnologías de la comunicación, la microelectrónica y la computación, productos en última instancia de conocimiento intensivo, tal como los patrones de consumo mucho más diferenciados e individualizados. "La estructura de la producción y el consumo tal como lo social, la clase, el género, y la división espacial del trabajo, están siendo modificados como resultado" (Martin, 1994). Para Swyngedouw (2004), la relación del trabajo y el Estado moderno fordista se funda en la lucha de los trabajadores que, al saltar las escalas propias de su espacio, trascienden sus lugares adquiriendo en el camino una cuota importante de poder.

Aceptar la posibilidad de una nueva época es a veces desorientador como lo manifiestan Gregory et al. (1994), ya que nos remite "a una impaciencia creciente al movernos más allá de los paradigmas teoréticos, metodológicos y epistemológicos del período de la postguerra". De acuerdo a ellos, la dramática reestructuración y desestabilización del capitalismo mundial, y el incremento de la globalización de la producción, las finanzas o incluso la cultura, son la cristalización de un nuevo orden. Smith (1994) señala, en lo que respecta a la cultura, el capital y el cambio urbano:

“El postmodernismo es una noción compleja y desafiada que es asociada con tendencias en el arte, la literatura y la arquitectura tal como con desarrollos en la filosofía y la teoría social. Es una condición cultural que, en ciudades, encuentra expresión en el medio ambiente construido, en la organización del consumo, en la conducta de la vida social y en la negociación de la identidad personal".

No obstante, Swyngedouw \& Kaïka (2003) resaltan que si bien es cierto que la modernidad fue la expresión cultural del orden social de la postguerra, la postmodernidad no sería más que la expresión cultural de las últimas décadas de un desarrollo intensificado del modernismo propiamente tal.

De cualquier manera, creemos estar atravesando una transformación mayor dentro de la trayectoria del desarrollo geopolítico y socioeconómico global. Esta investigación en parte da cuenta de eso. Collico, antiguo barrio industrial de la ciudad de Valdivia, se manifiesta como un caso de estudio donde observamos la convergencia de diversos modelos de construcción del espacio urbano. De esta manera, el objetivo general de nuestra investigación fue establecer la vinculación de los distintos paradigmas, surgidos desde el período moderno hasta el postmoderno, y analizar la evolución de la forma urbana en los distritos industriales de las ciudades de tamaño medio, estableciendo ciertos elementos singulares propios del caso de estudio.

\section{Paradigmas del mundo moderno y postmoderno: el caso de Collico}

Creemos relevante avanzar debatiendo acerca de la dimensión espacial de las ideologías y de cómo los modelos económicos y políticos se manifiestan en la dinámica urbana. En este sentido, Lefebvre (1991) convencido del fuerte vínculo entre las ideologías y el espacio, reflexiona " ¿Qué es una ideología sin un espacio a la cual referirse, un espacio que la describa, cuyo vocabulario y nexos hacen uso de ella y cuyos códigos esta abraza?"

La transición hacia un paradigma nuevo, entendido como un modelo o patrón acepta- 
do (Kuhn, 2000), contiene siempre un período de crisis que desemboca en consecutivas y radicales transformaciones. Neffa (1998) indica que:

"Por eso, un sistema viable y coherente en un viejo paradigma, encuentra por supuesto dificultades considerables para evolucionar en dirección de los nuevos principios. En consecuencia una vez establecido, un sistema productivo deviene estructuralmente estable y bloquea las innovaciones portadoras de un sistema considerado superior, incompatibles con las formas de coordinación propias del viejo sistema".

De acuerdo a Méndez y Caravaca (1999) cada etapa de crecimiento industrial ha estado acompañada de un modelo de organización dominante, constituida por un paradigma tecnológico, un régimen de acumulación, un modo de regulación y un modelo territorial coherente con cada etapa. No obstante, si bien es cierto que, en términos generales, en la actualidad podemos presenciar una transformación en este sentido, para Santos (2000) la historia del capitalismo puede ser dividida en períodos, "pedazos de tiempo marcados por cierta coherencia entre sus variables significativas, que evolucionan diferentemente, más dentro de un sistema". De acuerdo a él, esto ha sido hasta ahora, ya que en el período actual coexisten las etapas con sus correspondientes crisis, superponiéndose ambas, de esta manera el proceso de crisis es permanente. En una conceptualización más precisa, para Castells (1995) la reestructuración productiva es:

"El proceso mediante el cual los modos de producción transforman sus medios organizativos para llegar a realizar los principios estructurales inalterables de su operación [...] y en el caso del capitalismo, el impulso que lleva al capital privado a maximizar beneficios constituye el motor fundamental del crecimiento, la gestión y el consumo".

Los procesos de reestructuración del sector productivo, propios del paradigma emergente, comprenden importantes costos sociales en términos de desempleo y de desaparición de sectores productivos. Swyngedouw \& Kaïka (2003) indican que en las últimas dos décadas se ha desencadenado un profundo proceso de reestructuración en todos los aspectos de la vida cotidiana, como también en el orden tecnológico, espacial, social y político de nuestras ciudades, lo que se manifiesta en una transformación espacial, percibida o no, dependiendo del vigor con el cual la reestructuración sea llevada a cabo.

Esto, como se ha dicho anteriormente, genera sin duda una evolución en la dinámica urbana, la cual responde a la desaparición de funciones no competitivas y a los procesos de contestación y reacción por parte de la comunidad al modelo existente desestructurador del espacio circundante. De esta manera, la zona industrial y los barrios aledaños son configurados por el paradigma en curso o por la crisis que este enfrenta. Por otra parte, Swyngedouw \& Kaïka (2003) establecen que lo que existe es una fragmentación interna y una integración externa al espacio económico global. Para ellos esto se traduce en una "glocalización". En este mismo sentido, De Mattos (2002a) explica que:

"Esto, sin embargo, no implica afirmar que de esta manera desaparece o se esfuma un conjunto de rasgos inherentes a la identidad básica de cada una de ellas; por el contrario, existe evidencia acerca de que en virtud de estos procesos cada ciudad se transforma, pero preservando muchos de los rasgos establecidos y consolidados a lo largo de su historia, que son los que la distinguen de otras ciudades de su mismo ámbito geográfico".

En definitiva, creemos que la ciudad se ajusta a estos nuevos paradigmas y sus consecutivas crisis, no obstante la comunidad no permanece estática ante las sucesivas reestructuraciones productivas que demanda el modelo. Swyngedouw (2004) sugiere que "el poder social que puede ser movilizado es dependiente de la escala o el nivel espacial en el cual los actores sociales operan", argumento compartido por González (2006).

Quienes habitan el vecindario reaccionan y resisten, construyendo pequeñas para- 
dojas urbanas que dan cuenta de una realidad paralela, que si bien es cierto muchas veces es imperceptible en el espacio, contribuye a otorgar sentido al lugar que ellos ocupan (Rodríguez, 2002).

\section{Marco metodológico}

En este estudio se utilizó una metodología cualitativa, entendida como una estrategia sistemática y empírica para responder a interrogantes acerca de la gente en contextos sociales particulares (Locke et al., 2000). Empleamos el estudio de caso por ajustarse mejor a esta investigación, donde la unidad de estudio corresponde a un barrio tradicionalmente definido como industrial. El barrio, como unidad de análisis, no permite establecer una generalización estadística (es decir desde la muestra al universo). No obstante, de acuerdo a Yin (1998) sí es posible una generalización analítica, es decir, utilizar un caso de estudio para ilustrar, representar y generalizar una teoría. La diversidad de evidencia, propia del caso de estudio, cumple también el objetivo de otorgar mayor confiabilidad en la información recolectada.

\section{Área de estudio}

La unidad territorial constituida como caso de estudio corresponde a un barrio industrial-residencial (dentro de la percepción de la gente) denominado Collico, inserto en el margen norte de la ciudad de Valdivia que en conjunto con la industria adyacente es distinguido como barrio industrial a partir de mediados del siglo XIX.

\section{Muestra}

Todos los residentes fueron considerados participantes dentro de la investigación por estar en el escenario natural de los fenómenos urbanos. No obstante, en este amplio espectro la muestra elegida para ser entrevistada o encuestada correspondió a todos los residentes del barrio de elite, algunos residentes seleccionados del barrio obrero que trabajaron o trabajan en las industrias actualmente, y otros que residen en el barrio sin ser trabajadores de las industrias en cuestión.
También se incorporaron antiguos dueños del barrio de elite, aun cuando actualmente no residan en el barrio debido al fenómeno de desplazamiento de los dueños de los medios de producción hacia otros sectores más acomodados de la ciudad. Para seleccionar a los entrevistados se utilizó la técnica de informantes claves, donde se le pedía a estos el nombre de otra persona con información relevante.

Las encuestas se llevaron a cabo durante el transcurso de tres días; cuatro integrantes del equipo de investigación realizaron el procedimiento, seleccionando aleatoriamente a la gente en la calle. Dichos participantes debían cumplir el requisito de residir o haber residido en el vecindario de Collico durante los últimos 50 años.

\section{Técnicas de recolección de información}

En primera instancia se utilizó la observación participante del lugar, la que se llevó a cabo durante los tres primeros meses de ejecución del proyecto, estableciéndose un tiempo de observación de dos horas semanales, lo cual permitió hacer un análisis espacial de los lugares ${ }^{3}$. Se tomaron fotografías y se elaboró un registro tanto de las estructuras de los lugares (diseño arquitectónico-urbanístico y funcionalidad), como de las prácticas urbanas que se desarrollaban. En segunda instancia se realizaron entrevistas con los integrantes de la muestra, de manera de recoger las historias locales y las percepciones de los vecinos acerca de las transformaciones surgidas en el contexto antes descrito. Este procedimiento se Ilevó a cabo mediante la entrevista en profundidad a residentes del barrio que entregaron información relevante acerca de otros individuos que trabajaron o trabajan en esta actividad o han vivido un número mayor a 50 años en este vecindario, sin haber necesariamente trabajado en las industrias de Collico. Las entrevistas se realizaron en un contexto en el cual se fue ahondando progresivamente en la historia del barrio, al mismo tiempo que se les iban mostrando a los consultados

3 El análisis espacial es entendido desde la metodología que se aplica tanto en el diseño arquitectónico como en la disciplina geográfica. 
fotografías y mapas de manera que pudieran identificar claramente en el espacio las ideas que estaban desarrollando. También se llevó a efecto una encuesta cerrada a 25 vecinos del sector, escogidos aleatoriamente a quienes se les preguntó acerca del estado actual y pasado del vecindario, en tópicos como equipamiento, servicios, trabajo, vivienda.

La información suplementaria fue recopilada a partir de fotografías antiguas tomadas en la zona de estudio, entregando una perspectiva histórica de los hechos urbanos acaecidos en los lugares de estudio. Para complementar, se recopilaron notas de diarios antiguos y de archivos históricos que dieran cuenta de la estructura del lugar y de su funcionalidad. Se recopilaron todas las notas periódicas de entre los años 1952 y 1984 que hablaran del vecindario. Adicionalmente se construyó la cartografía del barrio para poder visualizar la dinámica urbana en los últimos 150 años.

\section{El vertiginoso tránsito de un barrio preindustrial a uno industrial}

Si bien es cierto que la etapa preindustrial marcada por un capitalismo mercantil había terminado a finales del siglo XVIII en Europa, se puede decir que en esta fase se imprimió, al menos al inicio, el carácter de hacer industria de los inmigrantes alemanes que se asentaron en Valdivia. Al inicio estos poseían una capacidad tecnológica muy reducida, que junto con la utilización de energías renovables tales como el agua y el uso intensivo de la madera, explican la fuerte dependencia del medio natural y la localización en los frentes fluviales y cercanos a los bosques (Otero, 2006).

Se usaban herramientas y maquinarias dependientes de la fuerza humana y de la tracción animal, lo que le otorgaba un cierto carácter manual a la producción. En esta etapa de incipiente industrialización, la estructura del trabajo industrial y artesanal de manufactura concentraba alrededor de un $10 \%$ a un $15 \%$ de la población en los centros urbanos, existiendo un incipiente nivel de concentración urbana en torno a la acti- vidad industrial. En el caso de Valdivia, de acuerdo a Alfonso (1900) en el año 1885 la población alcanzó a 23.531 habitantes y el porcentaje de población urbana era de un $38 \%$. En esta fecha 3.000 artesanos se desempeñan en distintas labores. De acuerdo al mismo autor, en 1894 el número de obreros trabajando en industrias era alrededor de 1.200 .

Collico (en mapudungun aguas rojas), antiguo barrio industrial de Valdivia, Región de Los Ríos, se originó en la ribera norte del río Callecalle. Aun cuando existen antecedentes históricos desde el siglo XVI (Guarda, 1950), el origen de la transformación desde una propiedad rural a un barrio preindustrial se aprecia recién hacia mediados del siglo XIX, con la colonización alemana encargada a Bernardo Philippi y Vicente Pérez Rosales (Cariola y Sunkel, 1982), en las provincias de Valdivia y Llanquihue.

Las zonas aledañas a los cursos fluviales -especialmente en el caso de Valdivia- fueron los sectores donde la industria tuvo una importante presencia en el desarrollo de nuevas zonas productivas. Esto se debió tanto a la facilidad para la obtención de la energía necesaria para el funcionamiento, como a la conectividad necesaria en el ingreso de las materias primas y en la salida de los productos manufacturados. Es así que la utilización de cursos de agua benefició enormemente el traslado de los productos, ya que estos eran conducidos por el río hasta el puerto de Corral, donde más tarde se exportaban directamente hacia Europa. De acuerdo a Stolzenbach y Schwarzenberg (c/ p), el molino Kunstmann poseía el vapor "Collico", y la curtiembre Stolzenbach el falucho denominado "Ceres", de manera de facilitar el traslado de la producción y los insumos a la industria.

No es extraño entonces que el arribo y asentamiento de los colonos alemanes al sector de Collico se concentrara en los tres canales, afluentes del río Callecalle, que más tarde Ilegarían a ser el eje principal del barrio (Av. Balmaceda). En la trama de 1890 es posible apreciar que el incipiente desarrollo urbano se encuentra vinculado a las industrias molineras y a la curtiembre, tal como se observa en la Figura $N^{0} 1$. 
Figura $\mathrm{N}^{\circ} 1$

COMPLEJO KUNSTMANN

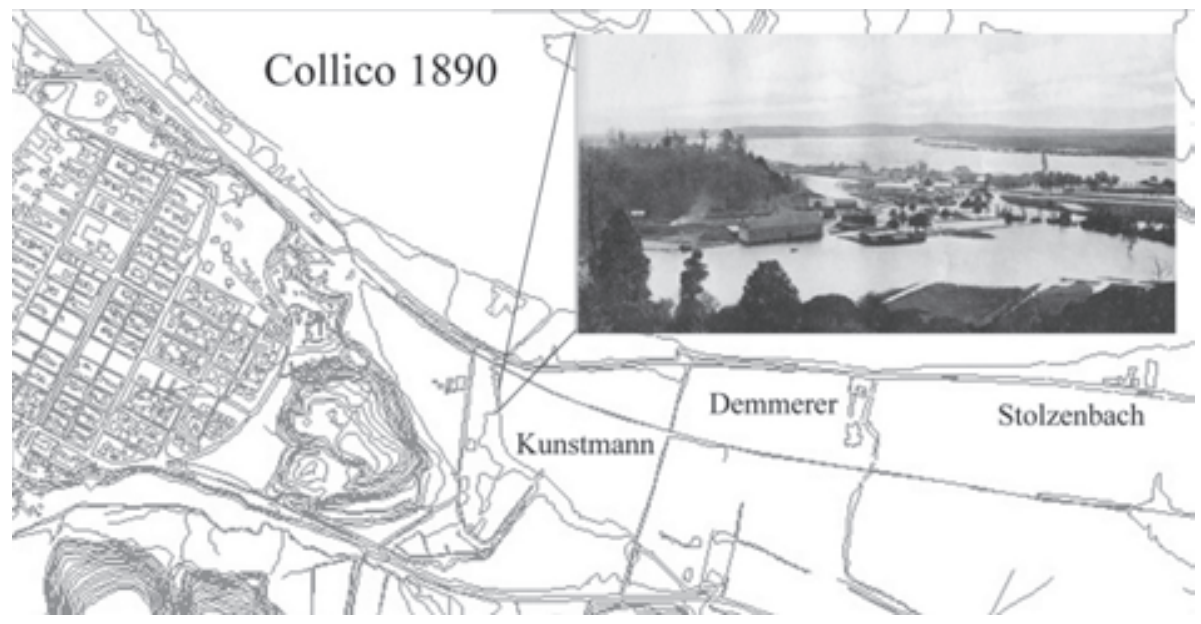

Fuente: Colección personal de los autores.

La familia Kunstmann, cuyo fundador fue Emmanuel Kunstmann von Lüttichau, se estableció en Collico en el año 1863, sector que para finales del siglo XIX gozaba de una floreciente empresa (Guarda, 2001). Esta situación fue un reflejo de un significativo capital inicial, según da cuenta Almonacid (1999). La construcción del pasaje Kunstmann, al frente del molino, donde vivían los principales miembros de la familia, es una demostración del capital familiar, inexistente en muchos otros industriales (Figura $\mathrm{N}^{\circ} 2$ ). Al final del pasaje estaba la entrada al fundo de 1.600 hectáreas, adquirido en 1867 (Almonacid, 1999), el que era explotado en actividades agrícolas (producción de trigo) y crianza de caballos. Por esta época las empresas localizadas en el incipiente poblado de Collico eran pequeñas y de carácter familiar, y mantenían una escasa división del trabajo (Bernedo, 1999).

Figura $\mathrm{N}^{\circ} 2$

PASAJE KUNSTMANN

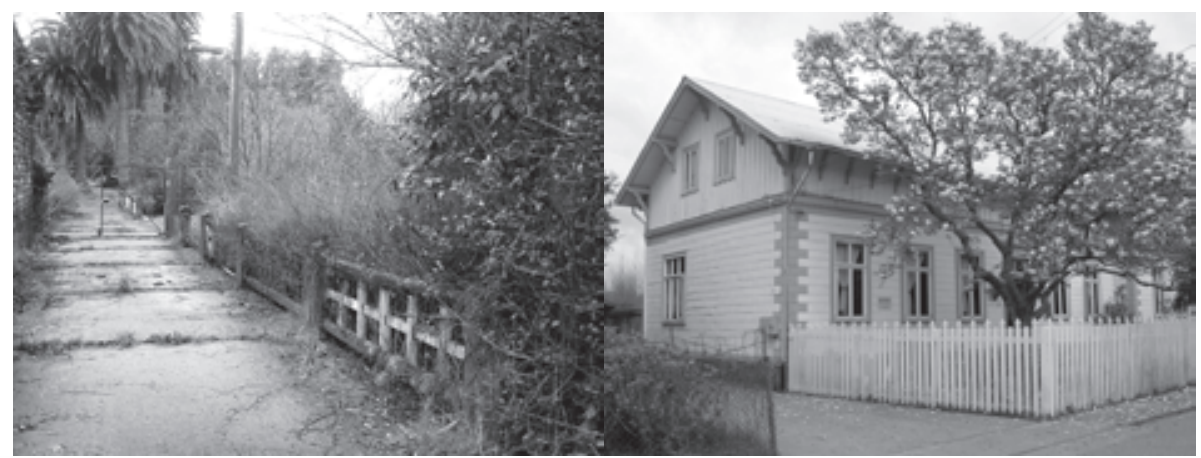

Fuente: Colección personal de los autores. 
Siguiendo por el eje principal, paralelo al río hacia el norte, se ubicó otra de las familias industriales, los Demerer, que arribaron en 1852 a Valdivia, estableciéndose inmediatamente en Collico y adquiriendo el terreno de la chacra de Mena (Guarda, 1995). Dentro del conjunto Demerer se encontraba el pasaje del mismo nombre, donde al final de este se emplazó el molino construido posteriormente (González, 2001). En menor escala que el complejo industrial Kunstmann, en 1854 funcionaba en el lugar una fábrica de aceite y sidra, un aserradero y un molino. A continuación hacia el norte por el lado del río se asentó la familia Stolzenbach, que Ilegó alrededor de 1880, fundando una curtiembre hacia el año 1887 (Almonacid, 1999).

En este período la actividad manufacturera era tremendamente diversificada, de manera de poder atender a la demanda local, no obstante, progresivamente se va expandiendo a mercados externos, de acuerdo a lo que se observa en el Cuadro $N^{\circ} 1$.

En Valdivia Ilegaron a existir en esa etapa 33 curtiembres, siendo uno de los más prósperos negocios en la ciudad. Hacer suela era muy conveniente porque compraban la materia prima (raulí y lingue) muy barata a los denominados cascareros. Bernedo (1999) establece al respecto de las curtidurías:
“[...] ya hacia la década de 1880, estaban organizadas como fábricas, es decir, como empresas modernas, que disponían de maquinaria movida por vapor y electricidad, que pagaban salarios en efectivo y que, en al menos un caso, aplicaban el concepto de productividad en este ámbito. En directa relación con la introducción de métodos productivos más eficientes, los empresarios del cuero lograron tempranamente penetrar con sus productos el mercado alemán en particular y el europeo en general. La importancia de este hecho fue subrayada en 1866 por el propio Intendente de la provincia $[\ldots]^{\prime \prime}$

Los industriales valdivianos pasaron rápidamente de esta etapa inicial al capitalismo industrial. Lo que a Europa le tomó en algunos casos 300 años, en Valdivia se dio en alrededor de 40 años, según lo señalado con anterioridad también por Gabriel Guarda en su libro Nueva Historia de Valdivia (2001).

Inmersos en la nueva lógica productiva, los industriales collicanos adquirieron nuevas maquinarias facilitadoras del proceso productivo. Es así que los Demerer tuvieron la primera fuente de energía eléctrica de CoIlico, una turbina hidroeléctrica que funcionaba gracias a las aguas del arroyo proveniente del cerro que da respaldo a Collico. Por otra parte, en 1910, de acuerdo a Almonacid (1999), el molino de piedra de la in-

Cuadro $\mathrm{N}^{\circ} 1$

PRODUCCIÓN DE LA ÉPOCA

\begin{tabular}{|c|c|c|}
\hline $\begin{array}{c}\text { Industrias existentes } \\
\text { en Valdivia }\end{array}$ & $\begin{array}{c}\text { Exportación a } \\
\text { puertos internacionales } \\
\text { (Ton) }\end{array}$ & Exportación a puertos nacionales \\
\hline $\begin{array}{l}2 \text { aguardientes } \\
2 \text { fábricas de cerveza } \\
6 \text { curtiembres } \\
1 \text { vapor de aserrar } \\
1 \text { fábrica de cepillar } \\
\text { maderas } \\
1 \text { fábrica de cola } \\
1 \text { fábrica de jabón }\end{array}$ & $\begin{array}{l}\text { Suelas: } 96,850 \\
\text { Maderas para } \\
\text { construcción: } 26,983 \\
\text { Salitre: } 5,418 \\
\text { Carne salada: } 2,211 \\
\text { Perú: } 30,430 \\
\text { Alemania: } 103,264 \\
\text { Total: } 133,694\end{array}$ & $\begin{array}{l}\text { Aguardiente, botas de becerro, carbón de } \\
\text { piedra, carne salada, cáscara de lingue, } \\
\text { cerveza, charqui, cola, crin en bruto, } \\
\text { grasa, jamones, lana común, maderas } \\
\text { para construcción, manteca de puerco, } \\
\text { mantequilla, quesos, suelas, trigo. } \\
\text { El total de lo exportado fue de } \$ 930.675\end{array}$ \\
\hline
\end{tabular}

Fuente: Tornero (1872). 
dustria Kunstmann fue sustituido por uno de cilindros, importado desde Dresden. De acuerdo a De Voz Eyzaguirre (1999), "símbolos máximos del progreso por su poder transformador, eran los ferrocarriles y la transformación de la energía para su aplicación en la producción". En este contexto Bernedo (1999) afirma:

“La solvencia económica de este grupo quedó en evidencia ya en el momento de su desembarco, cuando comenzaron a adquirir costosas propiedades urbanas y agrícolas en la ciudad y sus alrededores, así como materiales de construcción, ganado, pequeñas embarcaciones, etc. En este mismo sentido, también resulta sintomático que muchos inmigrantes, a poco de su llegada, se hicieron enviar desde Alemania herramientas y maquinarias, entre las que se contaban motores de vapor".

El desarrollo tecnológico que permitió la utilización de la máquina a vapor, la revolución de los transportes, la sustitución del trabajo manual por la mecanización, el trabajo en serie y la expansión de los mercados, permitió que la población vinculada a la industria creciera en un $30 \%$ en los centros urbanos.

En este sentido, las curtiembres contribuyeron en Valdivia significativamente a este crecimiento, ya que según Bernedo (1999) hacia 1900 eran las más grandes empleadoras de toda la región. No obstante, hacia 1906 empezaron a perder participación en el mercado alemán y para 1914 solo quedaban en pie dos curtiembres, entre ellas la ubicada en Collico (Stolzenbach c/p).

Las innovaciones incorporadas al transporte permitieron la ampliación de las áreas de abastecimiento y demanda y la consiguiente aglomeración urbana en torno a la industria, situación que se observa en general en el país (Borsdorf, 2003). Según el Cuadro $\mathrm{N}^{\circ} 2$ es posible observar que para esta fecha ya se contaba con un significativo contingente de gente viviendo en Collico. Sin embargo, el cuadro citado muestra también una cierta inestabilidad demográfica. De acuerdo al Cuadro $N^{\circ} 3$, el número de empleados en el sector no era verdadera- mente importante. De existir información de la cantidad de obreros que trabajaban en la industria Kunstmann en esta fecha, podría haber notables variaciones del cuadro señalado.

Cuadro $\mathrm{N}^{\circ} 2$

HABITANTES DE COLLICO

\begin{tabular}{|cc|}
\hline $\begin{array}{c}\text { Número de } \\
\text { habitantes de Collico }\end{array}$ & Año \\
\hline 1.294 & 1895 \\
1.254 & 1907 \\
\hline
\end{tabular}

Fuente: Espinoza (1897).

Cuadro $\mathrm{N}^{\circ} 3$

INDUSTRIAS UBICADAS EN COLLICO

\begin{tabular}{|lc|}
\hline \multicolumn{1}{|c|}{ Industria } & $\begin{array}{c}\text { Número de } \\
\text { empleados }\end{array}$ \\
\hline $\begin{array}{l}\text { Fábrica de jabón de } \\
\text { Enrique Koch } \\
\text { Curtiembre de Stolzenbach }\end{array}$ & 8 \\
$\begin{array}{l}\text { Fábrica de Cola de Alfredo } \\
\text { Kunstmann }\end{array}$ & 3 \\
$\begin{array}{l}\text { Curtiduría y molino de J. } \\
\text { Kunstmann } \\
\text { Curtiduría de Holl y } \\
\text { Molldenhavuer }\end{array}$ & $\mathrm{S} / \mathrm{i}$ \\
\hline
\end{tabular}

S/i: Sin Información.

Fuente: Alfonso (1900).

En este período el Estado comienza a aplicar medidas arancelarias a los artículos de importación, potenciando el crecimiento de la industria nacional. Con la recién estrenada Política de Industrialización para la Sustitución de Importaciones, el Estado estimulaba una evolución hacia un paradigma industrializador, de acuerdo a lo estipulado por De Voz Eyzaguirre (1999). En este sentido, Bernedo (1999) expresa que la Cámara Industrial de Valdivia (fundada en 1909), la segunda cámara después de la de Valparaíso, se abocó a presionar al gobierno para la 
protección de los intereses de las industrias locales y nacionales, insistiendo en que el gobierno debía fijar tarifas aduaneras especiales.

En una segunda reestructuración del sistema productivo se produjo una revolución tecnológica con la incorporación de nuevas fuentes de energía (hidrocarburos y electricidad), disminuyendo con esto el costo de producción y por supuesto aumentando notablemente la capacidad de carga. Se daba inicio entonces a la progresiva mecanización, produciendo en serie y en gran escala. En 1914 el molino construye una destilería de alcoholes, base sobre la cual es emplazada en 1922, la primera fábrica de levaduras de Chile, de acuerdo a la historia que aparece en el sitio web del molino ${ }^{4}$.

Conforme a una historia del molino Kunstmann recogida el año 1983 por el diario El Correo de Valdivia, se establecía que:

“En 1920 se duplicó la capacidad del molino e instaló una planta eléctrica propia con locomóvil de 300 caballos de fuerza. En esos años se agregó al molino

4 Disponible en: http://www.collico.cl/historia.shtml un destilatorio de alcoholes de grano con el que se proveía a grandes fábricas de alcohol con esta base. La destilería se conservó hasta 1960 cuando el sismo destruyó las instalaciones, lo que obligó a desmontarlas. Hasta 1960 funcionó asimismo la fábrica de fideos que también se terminó a causa del sismo y la situación económica posterior" (El Correo de Valdivia, 21/02/1982).

La creciente necesidad de inversiones en equipamientos y tecnología aceleró un proceso de concentración del capital, el cual es reflejado en la construcción de edificios industriales como el de la Figura $N^{\circ} 3$ correspondiente a la curtiembre Stolzenbach (1920). Colindante a esta se localiza la vivienda de la familia Stolzenbach, dueños de la industria.

Junto con el desarrollo del régimen de acumulación fordista, la fuerza de trabajo se organizó y aparecieron los primeros sindicatos, forzando con esto a las empresas y al Estado a mejorar las condiciones existentes. En el diario El Correo de Valdivia (4/9/1966) aparece una noticia que da cuenta del $37^{\circ}$ aniversario de la creación del sindicato Kunstmann con fecha 5 de septiembre de 1929. El mismo año 1966 aparece una noticia, el día 12 de noviembre, que da cuenta

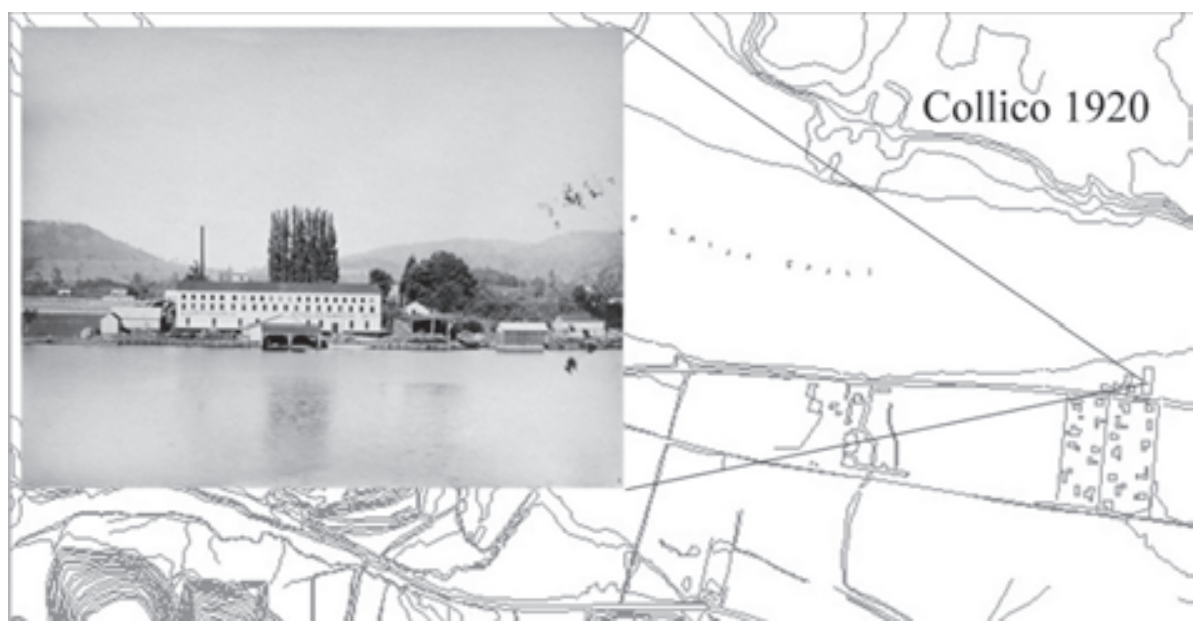

Fuente: Colección personal de los autores. 
del poder que ejerce el sindicato exigiendo una serie de mejoras salariales y de condiciones de trabajo, entre otras demandas.

En este período también crece el poder de intervención de los poderes públicos en la economía, creando empresas en sectores estratégicos para el desarrollo del país. La progresiva concentración de infraestructura, servicios, equipamientos sociales y viviendas para los trabajadores contribuyeron a una mayor densificación del sistema urbano como se observa en las Figuras $N^{\circ} 3$ y 4 . En estas figuras se aprecia cómo la transformación de paradigma del desarrollo hacia fuera, presente desde el siglo anterior, era sustituida por el de desarrollo hacia adentro (Sustitución de Importaciones) (De Mattos, 2002 b). Esto impactó profundamente en un proceso de fuerte urbanización, naciendo los barrios marginales periféricos. La industrialización y el poder estatal en la economía y la planificación, fueron los motores del desarrollo urbano (Borsdorf, 2003).

Por esta época existía la convicción en la ciudad de Valdivia de que los industriales eran quienes debían construir casas para los obreros (Almonacid, 2000). Circunscrita en la lógica del paternalismo industrial, esta idea cobró fuerza durante algún tiempo, dando paso posteriormente a un sistema de seguridad social supervisado por el Estado (Neffa, 1998). De esta manera dos empresas de Collico construyeron viviendas para sus trabajadores. En el caso de los molinos Kunstmann, las viviendas se ubicaron atrás de las casas vistas en la Figura $N^{0} 4$ (P. Kunstmann, c/p; Schwarzenberg, c/p). Posteriormente, en 1939 la empresa cedió parte del terreno para construir el equipamiento de bomberos del vecindario. De acuerdo a lo que se expresa en El Correo de Valdivia (22/3/1974), estas viviendas de los trabajadores fueron entregadas años después a sus moradores, relocalizándose en otros lugares. El concepto de paternalismo industrial permeaba a la sociedad entera y es así que la industria Stolzenbach también construyó, al frente de la industria, viviendas para sus obreros en el callejón del mismo nombre.

Dentro de la reestructuración correspondiente a esta época, los técnicos adquirieron un mayor nivel de protagonismo y ambas industrias tomaron bajo su responsabilidad la construcción de viviendas para los denominados "técnicos a cargo". Estas viviendas eran entregadas a trabajadores de mando medio. Las residencias de los técnicos de la industria Kunstmann se pueden observar en la Figura $N^{\circ} 4$. En la Figura $N^{0} 5$ se aprecia la casa entregada al técnico Schumacher por

Figura $\mathrm{N}^{\circ} 4$

AV. BALMACEDA

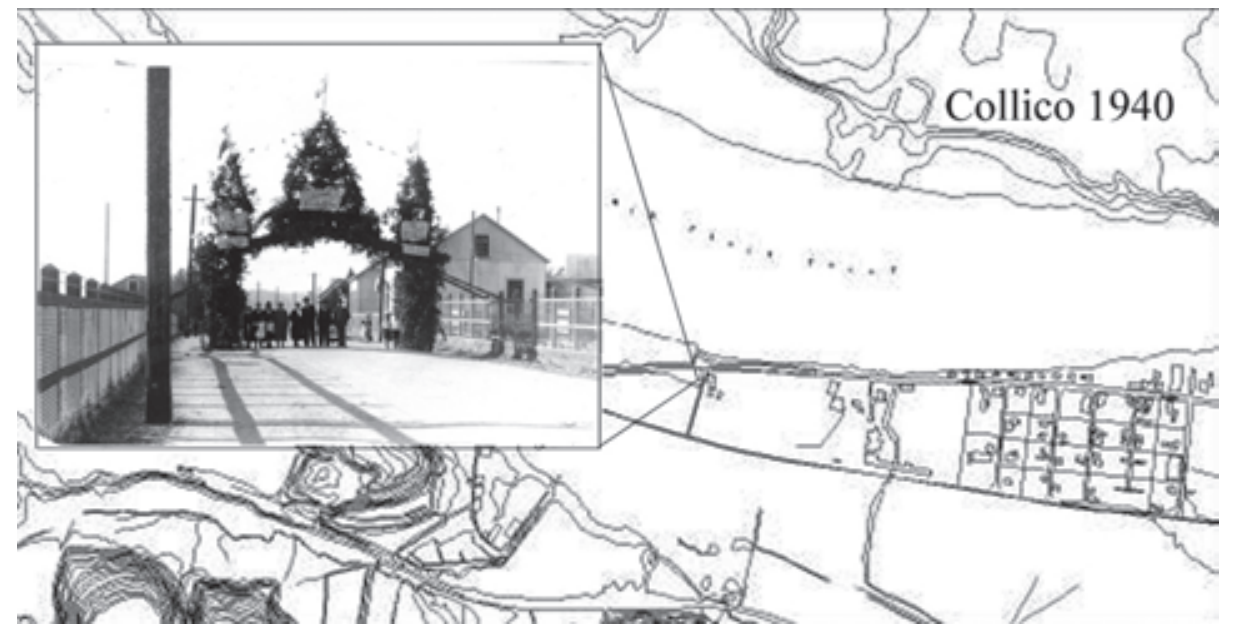

Fuente: Colección personal de los autores. 
Figura $\mathrm{N}^{\circ} 5$

RESIDENCIA ORIGINAL DEL TÉCNICO DE LA CURTIEMBRE

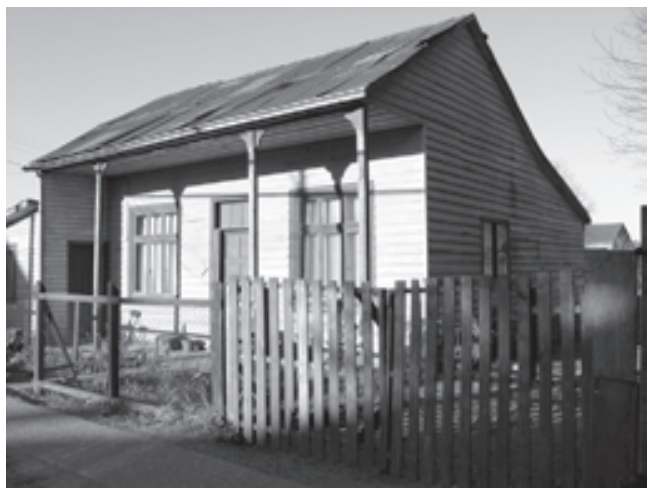

Fuente: Colección personal de los autores.

parte de la industria Stolzenbach, en los años 60. Esta vivienda está localizada al frente de la industria, cruzando la Avenida Balmaceda.

\section{El ocaso del barrio Collico}

El deterioro de las industrias de Collico se hizo visible con el violento terremoto de 1960, cataclismo que asoló la región destruyendo algunos sectores de la ciudad de Valdivia. Los ríos cambiaron su curso, nuevos cuerpos de agua nacieron, y la geografía se modificó marcadamente. En el complejo industrial Kunstmann desapareció gran parte del equipamiento original que daba lugar a la crianza de ganado y de caballos, junto con la chichería y la fábrica de cola. También el fundo que alimentaba de trigo al molino cambió de giro para convertirse progresivamente en un predio de explotación forestal, disminuyendo con esto el número de trabajadores (Miranda, c/p). La explicación de esto se puede encontrar en la puesta en vigencia del Decreto 701 (1976), que bonificaba las plantaciones forestales, lo cual contribuyó a transformar el fundo de agrícola en forestal.

El terremoto afectó además las vías de comunicación de la zona, destruyendo caminos. Valdivia estuvo muchos meses aislada, la inundación (Figura $N^{\circ} 6$ ) determinó que la industria Collico fuera desplazada por otras industrias en la producción de levaduras. Por otro lado, la industria Stolzenbach contaba con cerca de 100 trabajadores en el año 1945, y el terremoto provocó que parte de la industria original se hundiera, sin posibilidad de recuperación física ni económica.

De acuerdo a los entrevistados vinculados a la administración de la industria Kunstmann de aquella época, el gobierno de la Unidad Popular dio un nuevo golpe al complejo industrial. Había un gran peligro de expropiación de las casas del pasaje, de propiedad del molino Kunstmann, y amedrentados por esta posible amenaza, dividieron el patrimonio entre los integrantes de la familia. Debido a esto la familia no siguió viviendo en las residencias amenazadas, trasladándose al frente, en el borde del río y en otros casos otros miembros de la familia se trasladaron hacia otros sectores de la ciudad, alejándose del barrio Collico (Schwarzenberg, c/p).

De los tres fundos que tenía la familia Kuntsmann, dos fundos trigueros fueron expropiados por la reforma agraria, política llevada a cabo por el gobierno anterior. La familia mantuvo, sin embargo, el fundo CoIlico, el cual era esencialmente forestal en ese momento (Kuntsmann, c/p).

Con el paso del tiempo, el sistema tecnológico no fue capaz de responder al encarecimiento del sistema productivo y a la saturación de la demanda, la cual se presentaba cada vez más diversificada, fragmentándose los mercados y desestabilizándose la competencia. El cambio desde el capitalismo monopolista al capitalismo global contenía en sí mismo la crisis que haría emerger una revolución tecnológica, permitiendo reducir los costos unitarios de producción y reduciendo el consumo de energía (Neffa, 1998). Con estas nuevas tecnologías las fases de producción serían segmentadas, dividiendo el producto en pequeñas partes que serían fabricadas en distintos lugares por pequeñas empresas, contrastando con la lógica anterior del gran capital.

De esta manera, el agotamiento del sistema fordista producido en el centro de los países desarrollados a partir de finales de los 
Figura $\mathrm{N}^{\circ} 6$

INUNDACIÓN DESPUÉS DEL TERREMOTO

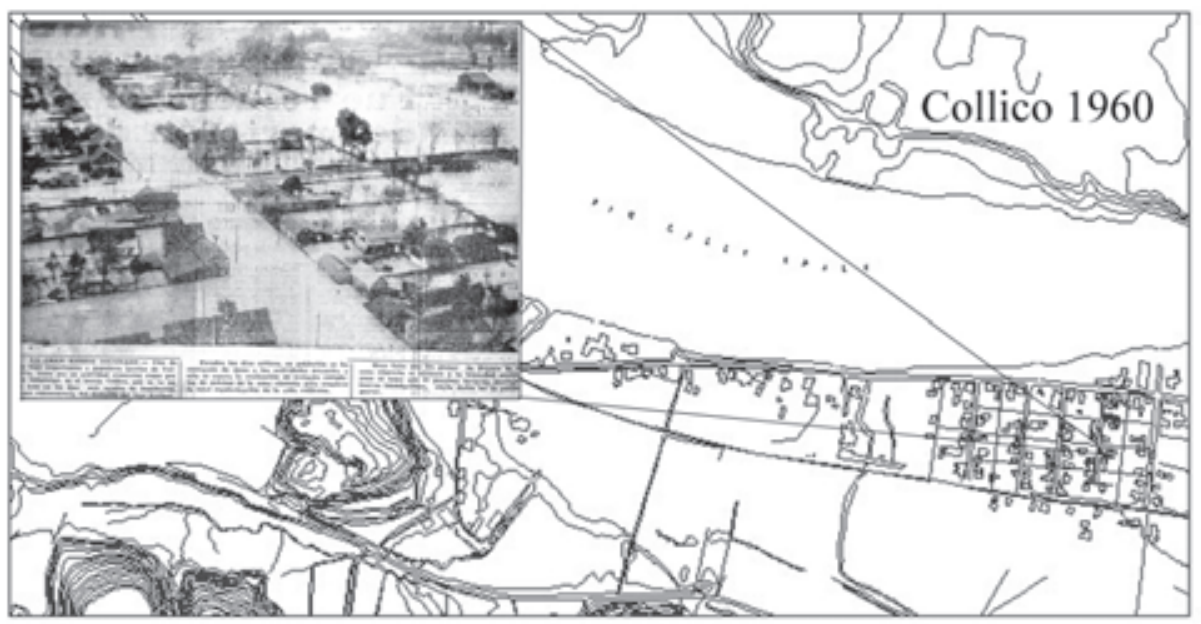

Fuente: Colección personal de los autores.

años 60, Ilega con veinte años de atraso al tercer mundo, de acuerdo a lo que Fuentes y Sierralta (2004) señalan. Existe un crecimiento negativo y una notable pérdida de empleos. En Latinoamérica la década de los 80 se denominó la década perdida, lo que dibujó una nueva geografía humana dentro de las ciudades, asentándose una atmósfera de desesperanza dentro de la ciudad y especialmente en este barrio.

A pesar de que las industrias del barrio Collico corresponden a lo que se conoce como industria de tecnología débil (Méndez y Caravaca, 1999), estas también se incorporarían a este modo de producción. Durante los años ochenta la empresa Kunstmann externalizó la mayoría de las actividades que antes desarrollaba al interior del complejo, y para el año 1993 se pone en marcha la asociación entre Levaduras Collico S.A. y la transnacional Burns Philp, adquirida el año 2004 por el grupo inglés $A B F(M$. Kunstmann, $c / p)$.

La industria Stolzenbach, después de quebrar económicamente, enajenando todo su patrimonio durante la década de 1980, se reabriría en abril del 2005 como Sociedad Industrial y Comercial Valdcuer, de propiedad del conglomerado alemán Lux Cuir $\mathrm{GMBH}$. Esta corporación alemana actualmente tiene interés en la materia prima, la cual posteriormente envía hacia Filipinas en donde se fabrican artículos de vestuario de lujosa terminación con destino al consumo europeo (Stolzenbach, c/p). La casa Demerer, parte del otrora sistema industrial de Collico, fue comprada en los años 80 por la familia Rojas Scheihing, instalándose una fábrica de muebles, la cual posteriormente a finales de los 90 pasaría a producir partes y a exportarlas (Carrasco, c/p).

La realidad industrial sometida a estos cambios produjo importantes transformaciones urbanas, reduciéndose la intervención del Estado en el ámbito laboral con la consiguiente desregulación del mercado del trabajo. En este sentido, Swyngedouw (2004) argumenta que "el proceso de producción glocalizada y las redes de interempresas no pueden estar separadas de los niveles de gobernanza glocalizada". Esto no podría desembocar más que en una importante pérdida de empleos, lo que algunos vinieron a denominar como la transición a la sociedad postindustrial, donde el sector de servicios tendría una mayor importancia en la economía. Caravaca y Méndez (2003) tienen una lectura distinta en este aspecto, según se señala a continuación:

"No asistimos, pues, a la transición desde una economía industrial a una econo- 
mía de servicios, sino de un tipo a otro de organización productiva que requiere mucha más cantidad de servicios inmateriales y de recursos intangibles, pero como soporte de una producción material que está lejos de haber perdido su importancia".

Caravaca y Méndez (2003) establecen que en áreas industriales tradicionales persisten algunas dedicadas al mercado local (talleres metalúrgicos, de artes gráficas, productos de alimentación, textiles). No obstante, la plusvalía de estas zonas hace peligrar su futuro, expulsando a la clase obrera y sus viviendas en detrimento de las oficinas y viviendas de lujo, como se puede observar en dos condominios cerrados construidos en el borde del río en los últimos 10 años dentro del barrio Collico. En la Figura $\mathrm{N}^{\circ} 7$ es posible advertir uno de estos condominios.

Por otro lado, con la creación del Ministerio de Vivienda y Urbanismo en la década de los años 60, el gobierno comienza también a asumir una mayor responsabilidad en el equipamiento público de los barrios y la planificación urbana en su conjunto (Hidalgo, 1999), estableciendo un nuevo paradigma urbano. De acuerdo a Hidalgo (1999):
"El papel del Estado sería más activo, dentro de sus principios se planteó que él debía ser el propietario del suelo, y el encargado de construir y controlar de acuerdo con las exigencias urbanísticas y sociales; las cuales debían primar sobre aquellas de carácter especulativo".

Hasta entonces la responsabilidad en la construcción de los barrios corría por cuenta de sus habitantes. Por ejemplo de acuerdo a M. Kunstmann (c/p), todavía en los años setenta no había alcantarillado (Figura $N^{\circ} 8$ ). El primer jardín infantil del vecindario se crea durante el gobierno de Eduardo Frei Montalva (1964-1970), según quien fuera la máxima autoridad de la provincia, Holzapfel (c/p). Durante esta misma década se construye la costanera con el fin de solucionar el problema recurrente de las inundaciones en el sector. Sumado a eso, el proyecto de pavimentación es abordado como una iniciativa mixta del Estado y los industriales del barrio, de acuerdo al diario El Correo de Valdivia (23/04/1967).

Con el golpe de Estado ocurrido en el año 1973, la política del suelo cambia repentinamente y el fisco se orienta más que nada a estimular el desarrollo inmobiliario, el cual es abordado por el privado. No obstante, con la recuperación de la democracia

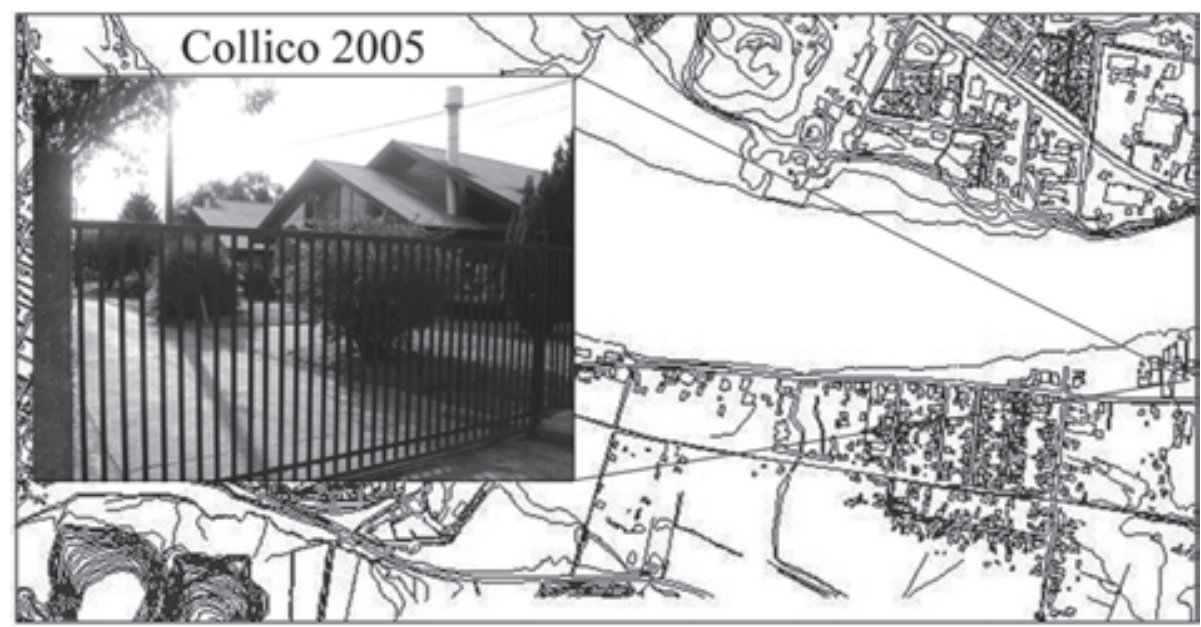

Fuente: Colección personal de los autores. 
Figura $\mathrm{N}^{\circ} 8$

ESTADO DEL VECINDARIO
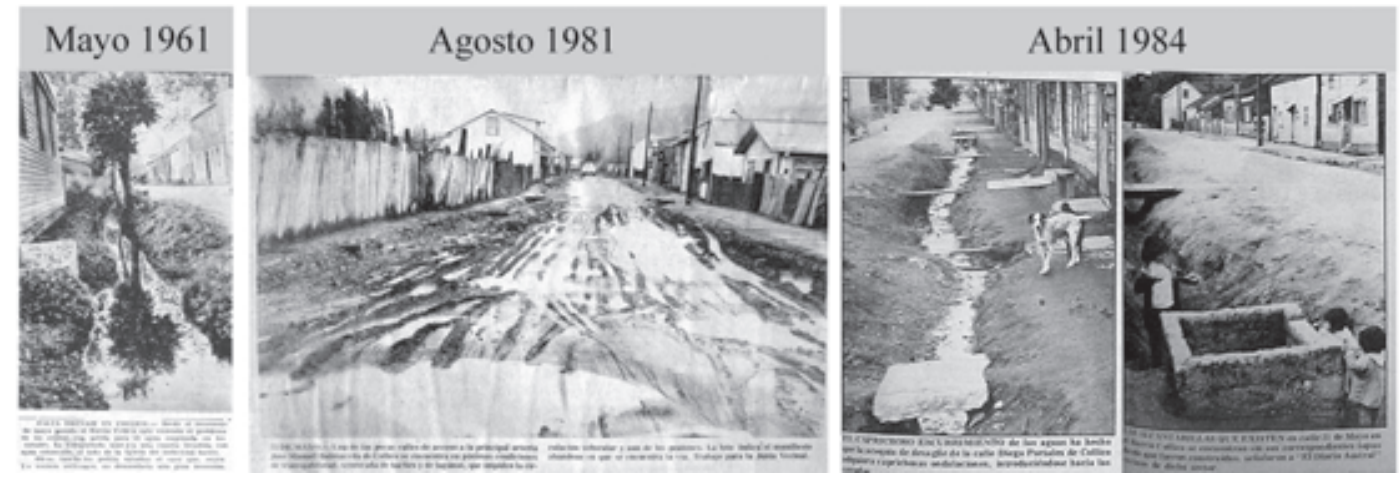

Fuente: El Correo de Valdivia $(1961,1981,1984)$.

Figura $\mathrm{N}^{\circ} 9$

MANIFESTACIONES DE LOS NUEVOS ADELANTOS
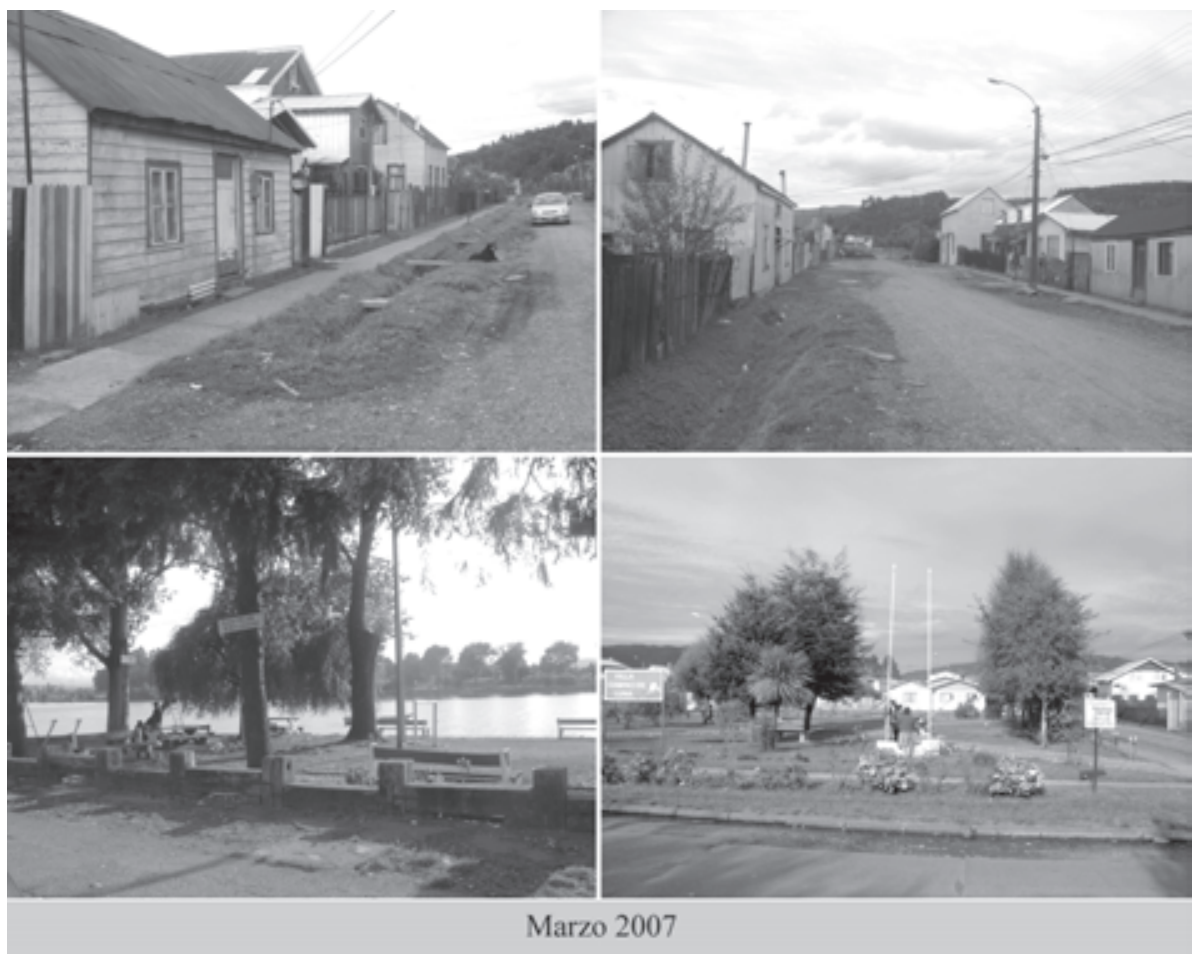

Fuente: Colección personal de los autores. 
en el año 1990, el Estado, a través de sus agencias, ha intentado retomar la labor de ordenador del espacio urbano, respondiendo a las aspiraciones de una mejor calidad de vida para sus habitantes (Sabatini y Arenas, 2000). Es así que en este vecindario industrial, donde el equipamiento público siempre fue precario, recién en los inicios del siglo XXI se proyectó la construcción de veredas, instalación de alcantarillado, electrificación, agua potable y la construcción de plazas (Figura No ${ }^{\circ}$ ).

\section{La resistencia en la conformación de un lugar}

Aquí se origina el punto de inflexión con el paradigma industrial postfordista. Aun cuando las tradicionales industrias se han adscrito al nuevo paradigma de acumulación flexible, los industriales vinculados al barrio Collico permanecen viviendo en el barrio. Esto contribuye a articular lazos de dependencia que fortalecen el sentido de pertenencia de los vecinos. El compromiso de los industriales se manifiesta en el fuerte vínculo de estos con los vecinos, quienes se refieren a sí mismos como parte de la comunidad de Collico. Este vínculo se establece mediante prácticas culturales propias del vecindario, como son las fiestas de entrega de regalos en época de navidad, construcción de naves para participar en la noche valdiviana. De acuerdo a lo manifestado en este tema, Schwarzenberg (c/p), actual dueño de los mollinos, Kunstmann reflexiona.

Para llegar a Valdivia desde aquí de la zona, había que tomar el barco porque no había forma de llegar, eso hizo que Collico creciera como un pueblo separado independiente con una identidad propia y su propio orgullo, y la empresa contribuyó a esto construyendo grandes poblaciones para sus obreros.

Pero en este aspecto, no podemos dejar pasar el hecho que de acuerdo a Swyngedouw \& Kaïka (2003) las fuerzas de la globalización y la fuerte competencia mundial han facultado a las elites a ser intensamente locales y globales, Ilegando a ser glocalizados.
Aún en estos tiempos, donde la gran estructura del capital irrumpe en las sociedades, Castells (1995) observa el fenómeno anteriormente descrito y considera que "también persiste un espacio de lugares en el que operan los agentes locales y se desarrollan la vida de las personas; y mientras esta última es cada vez más local, el espacio de flujos pasa a ser cada vez más global".

Por otra parte, los vecinos, antaño vinculados laboralmente a las industrias, presentan un gran sentido de arraigo con su vecindario, aun cuando muchos de ellos no se desempeñen ya en las empresas. Según las encuestas, los collicanos perciben en general su barrio en buen estado y aprecian bastante los vínculos intangibles generados a partir de los años, aunque la carencia de infraestructura y equipamiento es evidente. Existe, no obstante, un nivel de insatisfacción respecto del empleo, la falta de plazas de trabajo se ha agudizado en los últimos 30 años, incrementando la población desempleada. De acuerdo a M. Kunstmann (c/ p), los collicanos son bien unidos, y este es un argumento recurrente al momento de entrevistar a los vecinos. El sentido que le han otorgado a su vecindario traspasa, en muchos casos, la lógica propia de su clase, incapacitándolos para levantar una conciencia de clase acerca del espacio urbano en general. Tal como Harvey (1989) establece:

"En general, los movimientos de la clase obrera son más eficaces para organizarse internamente y dominar el lugar, que para controlar el espacio".

En este sentido, Swyngedouw y Kaïka (2003) refuerzan la idea que las regiones urbanas, más que nunca se han convertido en "paisajes del poder", donde islas de extrema riqueza y poder social son traspasados por lugares deprivados, excluidos y en declinación. Esto por supuesto ha sido de gran interés para el estudio de la geografía que de acuerdo a McDowell (1994) en su búsqueda por múltiples discursos acerca del sentido de lugar y la identidad, ha descubierto nuevas visiones del paisaje construido por los sin poder más que por los poderosos. 


\section{Consideraciones finales}

El barrio de Collico presenta transformaciones en su morfología derivadas de cambios paradigmáticos acontecidos en el ámbito económico, político y de trastornos ocasionados por el embate de la naturaleza. En una primera etapa, la incipiente urbanización se desarrolla en torno a los recursos naturales como canales, el río y el campo. Posteriormente, la tecnología propia del modelo industrial estimula un crecimiento poblacional aledaño a las industrias, provocando el crecimiento de la población obrera. A partir de esto los industriales junto con los obreros, contribuyen a forjar una identidad propia del barrio, construyendo viviendas para sus trabajadores. Estas modificaciones no afectan al barrio en su conjunto, sino que pueden ser percibidas en pequeños elementos que van, en conjunto, modificando el barrio en términos generales. Estas modificaciones se relacionan con la incorporación incipiente de equipamiento otorgado por los industriales y las demandas propias de los vecinos.

Los agentes modeladores del vecindario son, sin lugar a dudas, las industrias y sus vecinos. Son los primeros arquitectos de una geografía urbana propia del lugar. El crecimiento poblacional evidenciado en los mapas se produce en condiciones precarias hasta finales del siglo XX. Posteriormente, $y$ como resultado de un cambio de orientación de las políticas públicas, es el Estado el que asume la responsabilidad de realizar transformaciones de mayor impacto en un plazo más breve de tiempo.

El traspaso lento pero progresivo de una sociedad industrial a postindustrial se observa en la pérdida de empleos manifestada por los vecinos. Al mismo tiempo comienzan a aparecer de forma incipiente comunidades cerradas, atraídas por el significado histórico del barrio. Por esta misma razón, se configura un barrio donde las estructuras son pasajeras y donde las permanentes son escasas, básicamente las industrias y algunas residencias de carácter patrimonial. A pesar de esto, la percepción de los habitantes del lugar se encuentra fuertemente identificada con un barrio al que le asignan un carácter de comunidad.

\section{Referencias bibliográficas}

ALFONSO, J. Un viaje a Valdivia la civilización alemana en Chile Santiago de Chile: Imprenta Moderna, 1900.

ALMONACID, F. Industria, industriales y propiedad agrícola en el departamento de Valdivia, 1814-1914. Valparaíso: Tesis de Magíster en Historia, Universidad Católica de Valparaíso, 1999.

ALMONACID, F. Ideas y proyectos en torno a la vivienda obrera en la ciudad de Valdivia, 1900-1941. Revista Austral de Ciencias Sociales, 2000, No 4, p. 81-114.

BERNEDO, P. Los industriales alemanes de Valdivia, 1850-1914. Revista de Historia, 1999, Vol. 27, p. 5-42.

BORSDORF, A. Cómo modelar el desarrollo y la dinámica de la ciudad latinoamericana. EURE, 2003, Vol. 29, No 86, p. 37-49.

CARAVACA, I. y MÉNDEZ, R. Trayectorias industriales metropolitanas: nuevos procesos, nuevos contrastes. EURE, 2003, Vol. 29, No 87 , p. 37-50.

CARIOLA, C. y SUNKEL, O. La historia económica de Chile 1830 y 1930. Madrid: Ediciones Cultura Hispánica del Instituto de Cooperación Iberoamericana, 1982.

CARRASCO, J. Consulta personal a propósito de la investigación. Valdivia, (c/p).

CASTELLS, M. La ciudad informacional, Madrid: Alianza, 1995.

CUTHBERT, A. The form of cities political economy and urban design. Oxford: Blackwell, 2006.

DE MATTOS, C. Santiago de Chile de cara a la globalización: ¿otra ciudad? Revista de sociología política, 2002a, No 19, p. 31-54.

DE MATTOS, C. Transformación de las ciudades latinoamericanas: ¿Impactos de la globalización? EURE, 2002b, Vol. 28, № 85, p. 5-10. 
DE VOZ EYZAGUIRRE, B. El surgimiento del paradigma industrializador de Chile (18751900). Santiago de Chile: DIBAM, 1999.

EL CORREO DE VALDIVIA. Artículo noticioso. El Correo de Valdivia, 1961, 3 de mayo.

EL CORREO DE VALDIVIA. Artículo noticioso. El Correo de Valdivia, 1966, 5 de septiembre.

EL CORREO DE VALDIVIA. Artículo noticioso. Correo de Valdivia, 1967, 23 de abril.

EL CORREO DE VALDIVIA. Artículo noticioso. Correo de Valdivia, 1974, 22 de marzo.

EL CORREO DE VALDIVIA. Artículo noticioso. El Correo de Valdivia, 1981, agosto.

CORREO DE VALDIVIA. Artículo noticioso. El Correo de Valdivia, 1982, 21 de febrero.

EL CORREO DE VALDIVIA. Artículo noticioso. El Correo de Valdivia, 1984, abril.

ESPINOZA, E. Geografía descriptiva de la República de Chile. Barcelona: Imprenta Encuadernación Barcelona, 1897.

FUENTES, L y SIERRALTA, C. Santiago de Chile, ¿ejemplo de una reestructuración capitalista global? EURE, 2004, Vol. 30, № 91, p. 7-28.

GONZÁLEZ, M. Los Chicos no creen, pero así era. Historia y Palabra de Adultos Mayores. Santiago de Chile: Ponencia presentada en el IV Congreso Chileno de Antropología, 2001.

GONZÁLEZ, S. Scalar narratives in Bilbao: A cultural politics of scales approach to the study of urban policy. International journal of urban and regional research, 2006, Vol. 30, $\mathrm{N}^{\circ} 4$, p. 836-857.

GREGORY, D.; MARTIN, R. \& SMITH, G. Introduction human geography, social change and social science. In: GREGORY, D.; MARTIN, R. \& SMITH, G. Human Geography, society, space and social science. London: Macmillan, 1994, p. 1-18.

GUARDA, G. Conjunto urbano histórico arquitectónico Valdivia XVIII-XIX Santiago de Chile: Fondo Histórico, 1950.
GUARDA, G. La tradición de la madera. Santiago: Ediciones Universidad Católica de Chile, 1995.

GUARDA, G. Nueva historia de Valdivia. Santiago: Ediciones Universidad Católica de Chile, 2001.

HARVEY, D. The Condition of Post-Modernity. London: Blackwell. 1990.

HIDALGO, R. La vivienda social en Chile: la acción del Estado en un siglo de planes y programas. Revista Scripta Nova Revista Electrónica de Geografía y Ciencias Sociales, 1999, Vol. 45, № 1. Disponible en Internet: http://www.ub.es/geocrit/sn-45-1.htm

HOLZAPFEL, J. Consulta personal a propósito de la investigación. Valdivia, (c/p).

KHUN, T. La estructura de las revoluciones científicas. Santiago de Chile: Fondo de Cultura Económica, 2000.

KOSTOF, S. The city assembled the elements of urban form through history. Boston, New York, London: Bulfinch Press, 1992.

LOCKE, L.; SPIRDUSO, W. \& SILVERMAN, S. Proposals that work a guide for planning dissertations and grants proposals. California: Sage Publications, 2000.

LEFEBVRE, H. The Production of Space. London: Blackwell, 1991.

MARTIN, R. Economic theory and human geography. In: GREGORY, D.; MARTIN, R. \& SMITH, G. Human Geography, society, space and social science. London: Macmillan, 1994, p. 21-53.

McDOWELL, L. The transformation of cultural geography. En: GREGORY, D.; MARTIN, R. \& SMITH, G. Human Geography, society, space and social science. London: Macmillan, 1994, p. 146-173.

MÉNDEZ, R. y CARAVACA, I. Organización industrial y territorio. Madrid: Editorial Síntesis, 1999.

MIRADA, A. Consulta personal a propósito de la investigación. Valdivia, (c/p). 
NEFFA, J. Los paradigmas productivos Taylorista y Fordista y su crisis. Buenos Aires: Editorial Lumen, 1998.

OTERO, L. La huella del fuego. Valdivia: LOM, 2006.

KUNSTMANN, M. Consulta personal a propósito de la investigación. Valdivia, (c/p).

KUNSTMANN, P. Consulta personal a propósito de la investigación. Valdivia, (c/p).

RODRÍGUEZ, L. Geo-politic in reshaping U.S. urban spaces. Syracuse: Master thesis, 2002.

SABATINI, F. y ARENAS, F. Entre el Estado y el Mercado: resonancias geográficas y sustentabilidad social en Santiago de Chile. Eure, 2000, Vol. 26, № 79, p. 95-113.

SANTOS, M. Por uma outra globalizaçäo do pensamento único à consciência universal. São Paulo: Editora Record, 2000.

SCHWARZENBERG, G. Consulta personal a propósito de la investigación. Valdivia, (c/p).
STOLZENBACH, E. Consulta personal a propósito de la investigación. Valdivia, (c/p).

SMITH, S. Urban geography in a changing world. In: GREGORY, D.; MARTIN, R. \& SMITH, G. Human Geography, society, space and social science. London: MacmiIlan, 1994, p. 232-251.

SWYNGEDOUW, E. \& KAÏKA, M. The making of "glocal" urban modernities exploring de cracks in the mirror. CITY, 2003, vol. 7, No 1, p. 5-21.

SWYNGEDOUW, E. Globalization or "glocalization"?, networks, territories and rescaling. Cambridge Review of International Affairs, 2004, Vol. 17, No 1, p. 25-48.

TORNERO, R. Chile ilustrado: guía descriptiva del territorio de Chile de las capitales de provincia. Valparaíso: Librerías y agencias del Mercurio, 1872.

YIN, R. The abridge versión of Case Study research. In: BICKMAN, L \& ROG, D.J. Handbook of applied social research methods. USA: Sage Publications, 1998, p. 229-259. 